\title{
Analysis of stress response genes in moss Dicranum scoparium
}

\author{
Mazina A.B. ${ }^{1,2 *}$, Leksin I.Y. ${ }^{1,2}$, Minibayeva F.V. ${ }^{1,2}$ \\ ${ }^{1}$ Kazan Federal University, Kazan, Russia \\ ${ }^{2}$ Kazan Institute of Biochemistry and Biophysics, FRC KazSC RAS, Kazan, Russia \\ *email:abmazina@gmail.com
}

Mosses are the ancient terrestrial organisms, representing a branch of the evolution of higher plants, where the haploid gametophyte dominates in the life cycle. They can survive in extremely adverse conditions. The mechanisms of plant stress resistance include the expression of genes encoding defence proteins, such as antioxidant enzymes, chaperones, transcription factors. In this work, the following genes were selected: Lea3, F-box, DBF1, PHR1, ATG8. The transcriptomic data (RNA-Seq) of the moss Dicranum scoparium were taken from the NCBI SRA database. The library quality score was checked using FASTQC, then trimming of the reads with Trimmomatic and de novo assembly of the transcriptome using Trinity. From the file with the collected transcripts, a database was created to search for target genes using BLAST+. Protein sequences of target stress genes of the mosses with annotated genomes were used as a request. In D. scoparium, the target stress genes were discovered and found to be present in single homologous sequences. We also analyzed the constituency of each gene for the moss. Expression of stress genes was studied after the moss was subjected to various abiotic stresses, such as heavy metals, temperature stress, prooxidant, photosynthesis inhibitor, dehydration, for 1 and $12 \mathrm{~h}$. The qPCR method revealed that the genes DBF1, F-box, PHRI are genes of a rapid response, while ATG8, Lea3 increased their expression only after prolonged exposure to stresses. Thus, the involvement of these genes in the stress response of Dicranum suggests their evolutionary importance in the survival of higher plants.

Acknowledgements: This work is supported by the RFBR (No. 20-04-00721). 